\title{
The Existence and Uniqueness of Positive Periodic Solutions for a Class of Nicholson-Type Systems with Impulses and Delays
}

\author{
Ruojun Zhang and Fuyun Lian \\ School of Mathematical Sciences, Ocean University of China, Qingdao 266100, China \\ Correspondence should be addressed to Ruojun Zhang; zhangru1626@sina.com
}

Received 27 March 2013; Accepted 9 July 2013

Academic Editor: Ming Mei

Copyright (C) 2013 R. Zhang and F. Lian. This is an open access article distributed under the Creative Commons Attribution License, which permits unrestricted use, distribution, and reproduction in any medium, provided the original work is properly cited.

By establishing the equivalence, respectively, to the existence and uniqueness of positive periodic solutions for corresponding delay Nicholson-type systems without impulses, some criteria for the existence and uniqueness of positive periodic solutions for a class of Nicholson-type systems with impulses and delays are established. The results of this paper extend some earlier works reported in the literature.

\section{Introduction}

As we know, in order to describe the dynamics of Nicholson's blowflies, Gurney et al. [1] proposed a mathematical model

$$
N^{\prime}(t)=-\delta N(t)+P N(t-\tau) e^{-a N(t-\tau)},
$$

where $N(t)$ is the size of the population at time $t, P$ is the maximum per capita daily egg production, $1 / a$ is the size at which the population reproduces at its maximum rate, $\delta$ is the per capita daily adult death rate, and $\tau$ is the generation time. Nicholson's blowflies model described by delay differential equation (1) belongs to a class of biological system, and more and more biology systems have attracted more attention because of their extensively realistic significance [2-6]. In particular, the effects of a periodically varying environment are important for evolutionary theory, as the selective forces on systems in a fluctuating environment differ from those in a stable environment. There have been some results in the literature of the problem of the existence of positive periodic solutions for Nicholson's blowflies equation [7-11].

Recently, in order to describe the models of marine protected areas and B-cell chronic lymphocytic leukemia dynamics which are examples of Nicholson-type delay differential systems, Berezansky et al. [12], Wang et al. [13], and Liu [14] studied the following Nicholson-type delay systems:

$$
\begin{aligned}
N_{1}^{\prime}(t)= & -\alpha_{1}(t) N_{1}(t)+\beta_{1}(t) N_{2}(t) \\
& +\sum_{j=1}^{m} c_{1 j}(t) N_{1}\left(t-\tau_{1 j}(t)\right) e^{-\gamma_{1 j}(t) N_{1}\left(t-\tau_{1 j}(t)\right)}, \\
N_{2}^{\prime}(t)= & -\alpha_{2}(t) N_{2}(t)+\beta_{2}(t) N_{1}(t) \\
& +\sum_{j=1}^{m} c_{2 j}(t) N_{2}\left(t-\tau_{2 j}(t)\right) e^{-\gamma_{2 j}(t) N_{2}\left(t-\tau_{2 j}(t)\right)},
\end{aligned}
$$

where $\alpha_{i}(t), \beta_{i}(t), c_{i j}(t), \gamma_{i j}(t)$, and $\tau_{i j}(t) \in C(R,(0, \infty))$, $i=1,2, j=1,2, \ldots, m$. For coefficients and delays that are constants, Berezansky et al. [12] presented several results for the permanence and globally asymptotic stability of system (2). Supposing that $\alpha_{i}(t), \beta_{i}(t), c_{i j}(t), \gamma_{i j}(t)$, and $\tau_{i j}(t)$ are almost periodic functions, Wang et al. [13] obtained some criteria to ensure that the solutions of system (2) converge locally exponentially to a positive almost periodic solution. Furthermore, Liu [14] established some criteria for existence and uniqueness of positive periodic solutions of system (2) by applying the method of the Lyapunov functional. 
However, besides delay effects, impulsive effects likewise exist widely in many evolution processes in which states are changed abruptly at certain moment of time, involving such fields as medicine and biology, economics, mechanics, electronics, and telecommunications. That was the reason for the development of the theory of impulsive differential equations. We refer the reader to the monographs [15-17]. In practical, Yan [18] provided the method for studying a class of impulsive differential equations by changing impulsive equations into corresponding equations without impulses.

Therefore, it is necessary and reasonable to consider impulsive effects on the existence and uniqueness of positive periodic solutions for Nicholson-type delay systems (2). However, to the best of our knowledge, there are few results of this problem. Thus, techniques and methods of existence and uniqueness of positive periodic solutions for system (2) with impulsive effects should be developed and explored.

In this paper, we consider a class of Nicholson-type systems with impulses and delays

$$
\begin{aligned}
y_{1}^{\prime}(t)= & -\alpha_{1}(t) y_{1}(t)+\beta_{1}(t) y_{2}(t) \\
& +\sum_{j=1}^{m} c_{1 j}(t) y_{1}\left(t-\tau_{1 j}(t)\right) e^{-\gamma_{1 j}(t) y_{1}\left(t-\tau_{1 j}(t)\right)}, \\
y_{2}^{\prime}(t)=- & \alpha_{2}(t) y_{2}(t)+\beta_{2}(t) y_{1}(t)+\sum_{j=1}^{m} c_{2 j}(t) y_{2} \\
& \cdot\left(t-\tau_{2 j}(t)\right) e^{-\gamma_{2 j}(t) y_{2}\left(t-\tau_{2 j}(t)\right)}, \quad t \geq 0, t \neq t_{k}, \\
y_{i}\left(t_{k}^{+}\right)= & \left(1+b_{k}\right) y_{i}\left(t_{k}\right), \quad i=1,2, k=1,2, \ldots,
\end{aligned}
$$

where $\alpha_{i}(t), \beta_{i}(t), c_{i j}(t), \gamma_{i j}(t), \tau_{i j}(t) \in C([0, \infty),(0, \infty)), i=$ $1,2, j=1,2, \ldots, m . \Delta y_{i}\left(t_{k}\right)=y_{i}\left(t_{k}^{+}\right)-y_{i}\left(t_{k}^{-}\right)$are the impulses at moments $t_{k}$.

In (3), we will use the following hypotheses:

$\left(A_{1}\right) 0<t_{1}<t_{2}<\cdots$ are fixed impulsive points with $\lim _{k \rightarrow \infty} t_{k}=\infty$;

$\left(A_{2}\right)\left\{b_{k}\right\}$ is a real sequence and $b_{k}>-1, k=1,2, \ldots$;

$\left(A_{3}\right) \alpha_{i}(t), \beta_{i}(t), c_{i j}(t), \gamma_{i j}(t), \tau_{i j}(t)$, and $\prod_{0<t_{k}<t}\left(1+b_{k}\right)$ are periodic functions with common periodic $\omega>0, i=$ $1,2, j=1,2, \ldots, m$, and $k=1,2, \ldots$.

Here and in the sequel we assume that a product equals unit if the number of factor is equal to zero. We will only consider the solutions of (3) with initial values given by

$$
y_{i}(s)=\varphi_{i}(s), \quad s \in[-\tau, 0], \varphi_{i}(0)>0,
$$

where $\varphi_{i}(s) \in C([-\tau, 0],[0, \infty))$, and $\tau=\max \left\{\tau_{i j}^{+}\right\}, \tau_{i j}^{+}=$ $\max _{0 \leq t \leq \omega} \tau_{i j}(t), i=1,2, j=1,2, \ldots, m$.

The organization of this paper is as follows. In Section 2, we introduce some notations, definitions, and lemmas. In Section 3, some new sufficient conditions ensuring the existence and uniqueness of positive periodic solutions of (3) are derived by changing (3) into a corresponding equation without impulses. We study the existence of positive periodic solutions for the corresponding equation without impulses by coincidence degree theory, and we study the uniqueness of positive periodic solutions for the corresponding equation without impulses by the Lyapunov function. Finally, some conclusions are drawn in Section 4.

\section{Preliminaries}

For the sake of convenience, throughout this paper, we adopt the following notations:

$$
\begin{array}{rlrl}
\alpha_{i}^{-} & =\min _{0 \leq t \leq \omega} \alpha_{i}(t), & \alpha_{i}^{+} & =\max _{0 \leq t \leq \omega} \alpha_{i}(t), \\
\beta_{i}^{-} & =\min _{0 \leq t \leq \omega} \beta_{i}(t), & \beta_{i}^{+} & =\max _{0 \leq t \leq \omega} \beta_{i}(t), \\
p_{i j}^{-} & =\min _{0 \leq t \leq \omega} p_{i j}(t), & p_{i j}^{+} & =\max _{0 \leq t \leq \omega} p_{i j}(t), \\
q_{i j}^{-} & =\min _{0 \leq t \leq \omega} q_{i j}(t), & q_{i j}^{+} & =\max _{0 \leq t \leq \omega} q_{i j}(t), \\
i=1,2, & =1,2, \ldots, m .
\end{array}
$$

Definition 1. A map $y(t)=\left(y_{1}(t), y_{2}(t)\right)^{T}:[-\tau, \infty) \rightarrow$ $(0, \infty) \times(0, \infty)$ is said to be a solution of $(3)$ on $[\tau, \infty)$ satisfying the initial value condition (4), if

(i) $y_{i}(t)$ is absolutely continuous on each interval $\left(0, t_{1}\right]$ and $\left(t_{k}, t_{k+1}\right], k=1,2, \ldots$;

(ii) for any $t_{k}, k=1,2, \ldots, y_{i}\left(t_{k}^{+}\right)$and $y_{i}\left(t_{k}^{-}\right)$exist, and $y_{i}\left(t_{k}^{-}\right)=y_{i}\left(t_{k}\right)$

(iii) $y_{i}(t)$ satisfies $(3), i=1,2$.

Under the previous hypotheses $\left(A_{1}\right)-\left(A_{3}\right)$, we consider the following delay differential equation without impulses:

$$
\begin{aligned}
x_{1}^{\prime}(t)= & -\alpha_{1}(t) x_{1}(t)+\beta_{1}(t) x_{2}(t) \\
& +\sum_{j=1}^{m} p_{1 j}(t) x_{1}\left(t-\tau_{1 j}(t)\right) e^{-q_{1 j}(t) x_{1}\left(t-\tau_{1 j}(t)\right)}, \\
x_{2}^{\prime}(t)= & -\alpha_{2}(t) x_{2}(t)+\beta_{2}(t) x_{1}(t) \\
& +\sum_{j=1}^{m} p_{2 j}(t) x_{2}\left(t-\tau_{2 j}(t)\right) e^{-q_{2 j}(t) x_{2}\left(t-\tau_{2 j}(t)\right)}, \quad t \geq 0,
\end{aligned}
$$

with initial condition (4)

$$
\begin{aligned}
& x_{i}(s)=\varphi_{i}(s), \quad \text { for } s \in[-\tau, 0], \\
& \varphi \in C([-\tau, 0],[0, \infty)), \quad \varphi_{i}(0)>0,
\end{aligned}
$$

where

$$
\begin{array}{r}
p_{i j}(t)=\prod_{t-\tau_{i j}(t) \leq t_{k}<t}\left(1+b_{k}\right)^{-1} c_{i j}(t), \\
q_{i j}(t)=\prod_{0<t_{k}<t-\tau_{i j}(t)}\left(1+b_{k}\right) \gamma_{i j}(t), \\
\quad i=1,2, j=1,2, \ldots, m .
\end{array}
$$


By a solution $y(t)$ of $(6)$ on $[-\tau, \infty)$, we mean that an absolutely continuous function on $[-\tau, \infty)$ satisfies $(6)$ for $t \geq 0$ and initial condition (4). Similar to the method of [18], we have the following.

Lemma 2. Assume that $\left(A_{1}\right)-\left(A_{3}\right)$ hold. Then

(i) if $x(t)=\left(x_{1}(t), x_{2}(t)\right)^{T}$ is a solution (or positive $\omega$ periodic solution) of (6) on $[-\tau, \infty)$, then $y(t)=$ $\left(\prod_{0<t_{k}<t}\left(1+b_{k}\right) x_{1}(t), \prod_{0<t_{k}<t}\left(1+b_{k}\right) x_{2}(t)\right)^{T}$ is a solution (or positive $\omega$-periodic solution) of (3) on $[-\tau, \infty)$;

(ii) if $y(t)=\left(y_{1}(t), y_{2}(t)\right)^{T}$ is a solution (or positive $\omega$-periodic solution) of $(3)$ on $[-\tau, \infty)$, then $x(t)=$ $\left(\prod_{0<t_{k}<t}\left(1+b_{k}\right)^{-1} y_{1}(t), \prod_{0<t_{k}<t}\left(1+b_{k}\right)^{-1} y_{2}(t)\right)^{T}$ is a solution (or positive $\omega$-periodic solution) of (6) on $[-\tau, \infty)$.

Proof. First, we prove (i). If $x(t)=\left(x_{1}(t), x_{2}(t)\right)^{T}$ is a solution (or positive $\omega$-periodic solution) of $(6)$ on $[-\tau, \infty)$, then it is easy to see that $y_{i}(t)(i=1,2)$ is absolutely continuous on each interval $\left(0, t_{1}\right]$ and $\left(t_{k}, t_{k+1}\right], k=1,2, \ldots$, and, for any $t \neq t_{k}$,

$$
\begin{aligned}
y_{1}^{\prime}(t)+ & \alpha_{1}(t) y_{1}(t)-\beta_{1}(t) y_{2}(t) \\
-\sum_{j=1}^{m} c_{1 j}(t) y_{1}\left(t-\tau_{1 j}(t)\right) e^{-\gamma_{1 j}(t) y_{1}\left(t-\tau_{1 j}(t)\right)} & \prod_{0<t_{k}<t}\left(1+b_{k}\right) x_{1}^{\prime}(t)+\alpha_{1}(t) \prod_{0<t_{k}<t}\left(1+b_{k}\right) x_{1}(t) \\
& -\beta_{1}(t) \prod_{0<t_{k}<t}\left(1+b_{k}\right) x_{2}(t) \\
& -\sum_{j=1}^{m} c_{1 j}(t) \prod_{0<t_{k}<t-\tau_{1 j}(t)}\left(1+b_{k}\right) x_{1} \\
& \cdot\left(t-\tau_{1 j}(t)\right) e^{-\gamma_{1 j}(t) \prod_{0<t_{k}<t-\tau_{1 j}(t)}\left(1+b_{k}\right) x_{1}\left(t-\tau_{1 j}(t)\right)} \\
= & \prod_{0<t_{k}<t}\left(1+b_{k}\right)\left[x_{1}^{\prime}(t)+\alpha_{1}(t) x_{1}(t)-\beta_{1}(t) x_{2}(t)\right. \\
& -\sum_{j=1}^{m} c_{1 j}(t) \prod_{t-\tau_{1 j}(t) \leq t_{k}<t}\left(1+b_{k}\right)^{-1} x_{1} \\
\cdot\left(t-\tau_{1 j}(t)\right) & \left.\cdot e^{-\gamma_{1 j}(t) \prod_{0<t_{k}<t-\tau_{1 j}(t)}\left(1+b_{k}\right) x_{1}\left(t-\tau_{1 j}(t)\right)}\right] \\
0<t_{k}<t & {\left[1+b_{k}\right)\left[x_{1}^{\prime}(t)+\alpha_{1}(t) x_{1}(t)-\beta_{1}(t) x_{2}(t)\right.}
\end{aligned}
$$

$$
\begin{aligned}
& -\sum_{j=1}^{m} p_{1 j}(t) x_{1}\left(t-\tau_{1 j}(t)\right) \\
& \left.\cdot e^{-q_{1 j}(t) x_{1}\left(t-\tau_{1 j}(t)\right)}\right]=0 .
\end{aligned}
$$

Similarly, we have

$$
\begin{aligned}
& y_{2}^{\prime}(t)+\alpha_{2}(t) y_{2}(t)-\beta_{2}(t) y_{1}(t) \\
& \quad-\sum_{j=1}^{m} c_{2 j}(t) y_{2}\left(t-\tau_{2 j}(t)\right) e^{-\gamma_{2 j}(t) y_{2}\left(t-\tau_{2 j}(t)\right)}=0 .
\end{aligned}
$$

On the other hand, for every $t_{k} \in\left\{t_{k}\right\}, i=1,2$,

$$
\begin{gathered}
y_{i}\left(t_{k}^{+}\right)=\lim _{t \rightarrow t_{k}^{+}} \prod_{0<t_{j}<t}\left(1+b_{j}\right) x_{i}(t)=\prod_{0<t_{j} \leq t_{k}}\left(1+b_{j}\right) x_{i}\left(t_{k}\right), \\
y_{i}\left(t_{k}\right)=\prod_{0<t_{j}<t_{k}}\left(1+b_{j}\right) x_{i}\left(t_{k}\right) .
\end{gathered}
$$

Thus, for every $k=1,2, \ldots$,

$$
y_{i}\left(t_{k}^{+}\right)=\left(1+b_{k}\right) y_{i}\left(t_{k}\right) .
$$

It follows from (9) and (12) that $x(t)$ is the solution (or positive $\omega$-periodic solution) of (3) corresponding to initial condition (4).

Next, we prove (ii). If $y(t)=\left(y_{1}(t), y_{2}(t)\right)^{T}$ is a solution (or positive $\omega$-periodic solution) of $(3)$ on $[-\tau, \infty)$, then $y_{i}(t)(i=1,2)$ is absolutely continuous on each interval $\left(0, t_{1}\right]$ and $\left(t_{k}, t_{k+1}\right]$, and in view of (12), it follows that, for any $k=1,2, \ldots, i=1,2$,

$$
\begin{aligned}
x_{i}\left(t_{k}^{+}\right) & =\prod_{0<t_{j} \leq t_{k}}\left(1+b_{j}\right)^{-1} y_{i}\left(t_{k}^{+}\right) \\
& =\prod_{0<t_{j}<t_{k}}\left(1+b_{j}\right)^{-1} y_{i}\left(t_{k}\right)=x_{i}\left(t_{k}\right), \\
x_{i}\left(t_{k}^{-}\right) & =\prod_{0<t_{j} \leq t_{k-1}}\left(1+b_{j}\right)^{-1} y_{i}\left(t_{k}^{-}\right) \\
& =\prod_{0<t_{j}<t_{k}}\left(1+b_{j}\right)^{-1} y_{i}\left(t_{k}\right)=x_{i}\left(t_{k}\right) .
\end{aligned}
$$

Equations in (13) imply that $x_{i}(t)$ is continuous on $[0, \infty)$. It is easy to prove that $x(t)$ is also absolutely continuous on $[0, \infty)$. Now, one can easily check that $x(t)=\prod_{0<t_{k}<t}\left(1+b_{k}\right)^{-1} y(t)$ is the solution (or positive $\omega$-periodic solution) of (6) corresponding to initial condition (4). This completes the proof.

Lemma 3. Assume that $\left(A_{1}\right)-\left(A_{3}\right)$ hold. Then every solution of (3) is defined and positive on $[-\tau, \infty)$. 
Proof. Clearly, by Lemma 2, we only need to prove that every solution of $(6)$ is defined and positive on $[-\tau, \infty)$. In order to show that, we only need to see Lemma 2.3 in [13].

In the next section, we will study the existence of positive periodic solution of system (3). The method to be used in this paper involves the applications of the Mawhin's continuous theorem of the coincidence degree theory. We introduce some concepts and results concerning the coincidence degree as follows. Let $X$ and $Z$ be real Banach spaces, let $L$ : Dom $L \subset$ $X \rightarrow Z$ be a linear mapping, and let $N: X \rightarrow Z$ be a continuous mapping. The mapping $L$ is called a Fredholm mapping of index zero if $\operatorname{dim} \operatorname{Ker} L=\operatorname{codim} \operatorname{Im} L<+\infty$ and $\operatorname{Im} L$ is closed in $Z$. If $L$ is a Fredholm mapping of index zero, there exist continuous projectors $P: X \rightarrow X$ and $Q: Z \rightarrow$ $Z$ such that $\operatorname{Im} P=\operatorname{Ker} L$; and $\operatorname{Ker} Q=\operatorname{Im} L=\operatorname{Im}(I-Q)$.

Lemma 4 (see [19]). Let $X$ be a Banach space. Suppose that $L: D(L) \subset X \rightarrow X$ is a Fredholm operator with index zero and $N: \bar{\Omega} \rightarrow X$ is L-compact on $\bar{\Omega}$ with $\Omega$ open bounded in $X$. Moreover, assume that all the following conditions are satisfied:

(i) $L x \neq \lambda N x$, for all $x \in \partial \Omega \cap D(L), \lambda \in(0,1)$; (ii) $Q N x \neq 0$, for all $x \in \partial \Omega \cap \operatorname{Ker} L$;

(iii) the Brouwer degree

$$
\operatorname{deg}\{Q N, \Omega \cap \operatorname{Ker} L, 0\} \neq 0 \text {. }
$$

Then equation $L x=N x$ has at least one solution in $\bar{\Omega}$.

For ease of exposition, let

$$
\begin{gathered}
\left|x_{i}\right|_{\infty}=\max _{0 \leq t \leq \omega}\left|x_{i}(t)\right|, \quad u(t)=\left(x_{1}(t), x_{2}(t)\right)^{T}, \\
\left|x_{i}\right|_{2}=\left(\int_{0}^{\omega}\left|x_{i}(t)\right|^{2}\right)^{1 / 2}, \quad i=1,2, j=1,2, \ldots, m .
\end{gathered}
$$

We denote $X$ as the set of all continuously positive $\omega$-periodic functions $x(t)$ defined on $[0, \infty)$ and denote

$$
\|x\|_{X}=\max \left\{\left|x_{1}\right|_{\infty},\left|x_{2}\right|_{\infty}\right\}
$$

Then, $X$ is a Banach space when it is endowed with the norm $\|x\|_{X}$.

$$
\text { For } u(t)=\left(x_{1}(t), x_{2}(t)\right)^{T} \in X \text {, Let }
$$

$$
(N u)(t)=\left(\begin{array}{l}
-\alpha_{1}(t) x_{1}(t)+\beta_{1}(t) x_{2}(t)+\sum_{j=1}^{m} p_{1 j}(t) x_{1}\left(t-\tau_{1 j}(t)\right) e^{-q_{1 j}(t) x_{1}\left(t-\tau_{1 j}(t)\right)} \\
-\alpha_{2}(t) x_{2}(t)+\beta_{2}(t) x_{1}(t)+\sum_{j=1}^{m} p_{2 j}(t) y_{2}\left(t-\tau_{2 j}(t)\right) e^{-q_{2 j}(t) x_{2}\left(t-\tau_{2 j}(t)\right)}
\end{array}\right),
$$

$$
\begin{gathered}
(L u)(t)=\left(x_{1}^{\prime}(t), x_{2}^{\prime}(t)\right)^{T}, \\
P u=Q u=\frac{1}{\omega} \int_{0}^{\omega} u(t) d t \\
=\left(\frac{1}{\omega} \int_{0}^{\omega} x_{1}(t) d t, \frac{1}{\omega} \int_{0}^{\omega} x_{2}(t) d t\right)^{T} .
\end{gathered}
$$

In view of (17) and (18), the operator equation

$$
L u=\lambda N u
$$

(19) is equivalent to the following:

$$
\left(\begin{array}{l}
x_{1}^{\prime}(t) \\
x_{2}^{\prime}(t)
\end{array}\right)=\lambda\left(\begin{array}{l}
-\alpha_{1}(t) x_{1}(t)+\beta_{1}(t) x_{2}(t)+\sum_{j=1}^{m} p_{1 j}(t) x_{1}\left(t-\tau_{1 j}(t)\right) e^{-q_{1 j}(t) x_{1}\left(t-\tau_{1 j}(t)\right)} \\
-\alpha_{2}(t) x_{2}(t)+\beta_{2}(t) x_{1}(t)+\sum_{j=1}^{m} p_{2 j}(t) y_{2}\left(t-\tau_{2 j}(t)\right) e^{-q_{2 j}(t) x_{2}\left(t-\tau_{2 j}(t)\right)}
\end{array}\right),
$$

where $\lambda \in(0,1)$.

Again from (17) and (18), it is not difficult to show that Ker $L=R^{2}, \operatorname{Im} L=\left\{u(t)=\left(x_{1}(t), x_{2}(t)\right)^{T} \in X, \int_{0}^{\omega} x_{1}(t) d t=\right.$ $\left.\int_{0}^{\omega} x_{2}(t) d t=0\right\}$ is closed in $X$, and $\operatorname{dim} \operatorname{Ker} L=\operatorname{codim} \operatorname{Im} L$.
From the definitions of continuous projectors $P$ and $Q$, we can easily to get

$$
\operatorname{Im} P=\operatorname{Ker} L, \quad \operatorname{Ker} Q=\operatorname{Im} L .
$$


It follows that the operator $L$ is a Fredholm operator with index zero. Furthermore, the generalized inverse (of $L$ ) $K_{P}$ : $\operatorname{Im} L \rightarrow D(L) \cap \operatorname{Ker} P$ reads as

$$
\begin{aligned}
& \left(K_{P} u\right)(t) \\
& =\left(\begin{array}{l}
\int_{0}^{t} x_{1}(s) d s-\frac{1}{\omega} \int_{0}^{\omega} \int_{0}^{t} x_{1}(s) d s d t \\
\int_{0}^{t} x_{2}(s) d s-\frac{1}{\omega} \int_{0}^{\omega} \int_{0}^{t} x_{2}(s) d s d t
\end{array}\right) \\
& \quad \text { for } u(t)=\left(\begin{array}{l}
x_{1}(t) \\
x_{2}(t)
\end{array}\right) \in \operatorname{Im} L .
\end{aligned}
$$

Therefore, it is easy to see from (17) and (23) that $N$ is $L$ compact on $\bar{\Omega}$, where $\Omega$ is any open bounded set in $X$.

\section{Main Results}

Theorem 5. Assume that $\left(A_{1}\right)-\left(A_{3}\right)$ hold. Moreover, the following condition is satisfied:

$$
\left(A_{4}\right) \widetilde{D}=\min \left\{\alpha_{1}^{-}-\beta_{2}^{+}, \alpha_{2}^{-}-\beta_{1}^{+}\right\}>0 .
$$

\section{Then (3) has at least one positive $\omega$-periodic solution.}

Proof. Based on Lemma 4, what we need to do is just to search for an appropriate open bounded subset $\Omega$ for applying Mawhin's continuous theorem. To do this, it suffices to prove that the set of all possible positive $\omega$-periodic solution of (6) is bounded.

Let $u(t)=\left(x_{1}(t), x_{2}(t)\right)^{T}$ be an arbitrary positive $\omega$ periodic solution of (6). Corresponding to the operator equation (21), we have

$$
\begin{array}{r}
x_{1}^{\prime}(t)=\lambda\left(-\alpha_{1}(t) x_{1}(t)+\beta_{1}(t) x_{2}(t)\right. \\
\left.\quad+\sum_{j=1}^{m} p_{1 j}(t) x_{1}\left(t-\tau_{1 j}(t)\right) e^{-q_{1 j}(t) x_{1}\left(t-\tau_{1 j}(t)\right)}\right), \\
x_{2}^{\prime}(t)=\lambda\left(\begin{array}{l}
- \\
\alpha_{2}(t) x_{2}(t)+\beta_{2}(t) x_{1}(t)
\end{array}\right. \\
\left.\quad+\sum_{j=1}^{m} p_{2 j}(t) x_{2}\left(t-\tau_{2 j}(t)\right) e^{-q_{2 j}(t) x_{2}\left(t-\tau_{2 j}(t)\right)}\right) .
\end{array}
$$

Multiplying $x_{1}(t)$ and the first formula of (24), and then integrating from 0 to $\omega$, we obtain

$$
\begin{gathered}
\int_{0}^{\omega} x_{1}^{\prime}(t) x_{1}(t) d t \\
=\lambda\left\{\int_{0}^{\omega}-\alpha_{1}(t) x_{1}^{2}(t) d t+\int_{0}^{\omega} \beta_{1}(t) x_{1}(t) x_{2}(t) d t\right. \\
+\sum_{j=1}^{m} \int_{0}^{\omega} p_{1 j}(t) x_{1}(t) x_{1}\left(t-\tau_{1 j}(t)\right) \\
\left.\cdot e^{-q_{1 j}(t) x_{1}\left(t-\tau_{1 j}(t)\right)} d t\right\}
\end{gathered}
$$

hence,

$$
\begin{aligned}
& \left|\int_{0}^{\omega} \alpha_{1}(t) x_{1}^{2}(t) d t\right| \\
& \leq\left|\int_{0}^{\omega} \beta_{1}(t) x_{1}(t) x_{2}(t) d t\right| \\
& \quad+\sum_{j=1}^{m}\left|\int_{0}^{\omega} p_{1 j}(t) x_{1}(t) x_{1}\left(t-\tau_{1 j}(t)\right) e^{-q_{1 j}(t) x_{1}\left(t-\tau_{1 j}(t)\right)} d t\right| .
\end{aligned}
$$

Furthermore, from the Hölder inequality and $e^{x}>x$ (or $x$. $e^{-x}<1$ ) for $x>0$, we have

$$
\begin{aligned}
& \alpha_{1}^{-}\left|x_{1}\right|_{2}^{2} \\
& \leq\left|\int_{0}^{\omega} \alpha_{1}(t) x_{1}^{2}(t) d t\right| \\
& \leq \beta_{1}^{+}\left(\int_{0}^{\omega}\left|x_{1}(t)\right|^{2} d t\right)^{1 / 2} \cdot\left(\int_{0}^{\omega}\left|x_{2}(t)\right|^{2} d t\right)^{1 / 2} \\
& \quad+\sum_{j=1}^{m} \int_{0}^{\omega} \mid p_{1 j}(t) x_{1}(t) \frac{q_{1 j}(t) x_{1}\left(t-\tau_{1 j}(t)\right)}{q_{1 j}(t)} \\
& \leq \beta_{1}^{+}\left|x_{1}\right|_{2} \cdot\left|x_{2}\right|_{2}+\sum_{j=1}^{m} \frac{p_{1 j}^{+}}{q_{1 j}^{-}} \int_{0}^{\omega}\left|x_{1}(t)\right| d t \\
& \leq \beta_{1}^{+}\left|x_{1}\right|_{2} \cdot\left|x_{2}\right|_{2}+\sum_{j=1}^{m} \frac{p_{1 j}^{+}}{q_{1 j}^{-}} \sqrt{\omega} \cdot\left|x_{1}\right|_{2} ;
\end{aligned}
$$

that is,

$$
\alpha_{1}^{-}\left|x_{1}\right|_{2} \leq \beta_{1}^{+}\left|x_{2}\right|_{2}+\sum_{j=1}^{m} \frac{p_{1 j}^{+}}{q_{1 j}^{-}} \sqrt{\omega} .
$$


Similarly, we have

$$
\alpha_{2}^{-}\left|x_{2}\right|_{2} \leq \beta_{2}^{+}\left|x_{1}\right|_{2}+\sum_{j=1}^{m} \frac{p_{2 j}^{+}}{q_{2 j}^{-}} \sqrt{\omega}
$$

Combining (28) and (29), we get

$$
\left(\alpha_{1}^{-}-\beta_{2}^{+}\right)\left|x_{1}\right|_{2}+\left(\alpha_{2}^{-}-\beta_{1}^{+}\right)\left|x_{2}\right|_{2} \leq \sum_{j=1}^{m}\left(\frac{p_{1 j}^{+}}{q_{1 j}^{-}}+\frac{p_{2 j}^{+}}{q_{2 j}^{-}}\right) \sqrt{\omega} \text {. }
$$

Since $\widetilde{D}=\min \left\{\alpha_{1}^{-}-\beta_{2}^{+}, \alpha_{2}^{-}-\beta_{1}^{+}\right\}>0,(30)$ implies that $\left|x_{1}\right|_{2}$ and $\left|x_{2}\right|_{2}$ are bounded. Therefore, according to the previous proof, there exists a positive constant $D_{1}$ (independent of $\lambda$ ) such that

$$
\left|x_{1}\right|_{2} \leq D_{1}, \quad\left|x_{2}\right|_{2} \leq D_{1}
$$

From (24), together with the Hölder inequality and $x e^{-x}<1$ for $x>0$, we can obtain

$$
\begin{aligned}
\int_{0}^{\omega}\left|x_{1}^{\prime}(t)\right| d t \leq & \alpha_{1}^{+} \int_{0}^{\omega}\left|x_{1}(t)\right| d t+\beta_{1}^{+} \int_{0}^{\omega}\left|x_{2}(t)\right| d t \\
& +\sum_{j=1}^{m} p_{1 j}^{+} \int_{0}^{\omega} \mid \frac{q_{1 j}(t) x_{1}\left(t-\tau_{1 j}(t)\right)}{q_{1 j}(t)} \\
& \cdot e^{-q_{1 j}(t) x_{1}\left(t-\tau_{1 j}(t)\right)} \mid d t \\
\leq & \alpha_{1}^{+} \sqrt{\omega}\left|x_{1}\right|_{2}+\beta_{1}^{+} \sqrt{\omega}\left|x_{2}\right|_{2}+\sum_{j=1}^{m} \frac{p_{1 j}^{+}}{q_{1 j}^{-}} \omega D_{2}, \\
\int_{0}^{\omega}\left|x_{2}^{\prime}(t)\right| d t \leq & \alpha_{2}^{+} \int_{0}^{\omega}\left|x_{2}(t)\right| d t+\beta_{2}^{+} \int_{0}^{\omega}\left|x_{1}(t)\right| d t \\
& +\sum_{j=1}^{m} p_{2 j}^{+} \int_{0}^{\omega} \mid \frac{q_{2 j}(t) x_{2}\left(t-\tau_{2 j}(t)\right)}{q_{2 j}(t)} \\
\leq & \alpha_{2}^{+} \sqrt{\omega}\left|x_{2}\right|_{2}+\beta_{2}^{+} \sqrt{\omega}\left|x_{1}\right|_{2}+\sum_{j=1}^{m} \frac{p_{2 j}^{+}}{q_{2 j}^{-}} \omega D_{3} .
\end{aligned}
$$

(31)-(32) imply that there exist two positive constants $\mu_{i}, i=$ 1,2 , such that

$$
\int_{0}^{\omega}\left|x_{i}^{\prime}(t)\right| d t \leq \mu_{i}, \quad i=1,2
$$

Equations in (31) imply that there exist two points $\xi_{i} \in[0, \omega]$, $i=1,2$, and two positive constants $d_{i}, i=1,2$, such that

$$
\left|x_{i}\left(\xi_{i}\right)\right| \leq d_{i}, \quad i=1,2 .
$$

Since, for $\forall t \in[0, \omega]$,

$$
\begin{aligned}
\left|x_{i}(t)\right|= & \left|x_{i}\left(\xi_{i}\right)+\int_{\xi_{i}}^{t} x_{i}^{\prime}(s) d s\right| \leq d_{i} \\
& +\int_{0}^{\omega}\left|x_{i}^{\prime}(t)\right| d t, \quad i=1,2,
\end{aligned}
$$

from (33), it follows that there exists a positive constant $\zeta$ such that

$$
\left|x_{i}\right|_{\infty} \leq \zeta, \quad i=1,2
$$

Clearly, $\zeta$ is independent of $\lambda$. Let $H^{*}=\zeta+C$, where $C>1$ is taken sufficiently large so that

$$
\widetilde{D} H^{*}>\sum_{j=1}^{m}\left(\frac{p_{1 j}^{+}}{q_{1 j}^{-}}+\frac{p_{2 j}^{+}}{q_{2 j}^{-}}\right)
$$

Now, we take $\Omega=\left\{u(t)=\left(x_{1}(t), x_{2}(t)\right)^{T} \in X,\|u\|_{X}=\right.$ $\left.\max \left\{\left|x_{1}\right|_{\infty},\left|x_{2}\right|_{\infty}\right\}<H^{*}\right\}$. This satisfied condition (i) of Lemma 4.

When $u(t)=\left(x_{1}(t), x_{2}(t)\right)^{T} \in \partial \Omega \bigcap \operatorname{Ker} L,\left(x_{1}(t), x_{2}(t)\right)^{T}$ is a constant vector $\left(x_{1}, x_{2}\right)^{T}$ in $R^{2}$ with $\|u\|_{X}=$ $\max \left\{\left|x_{1}\right|_{\infty},\left|x_{2}\right|_{\infty}\right\}=H^{*}$, then

$$
Q N\left(\begin{array}{l}
x_{1} \\
x_{2}
\end{array}\right)=\left(\begin{array}{l}
-\frac{x_{1}}{\omega} \int_{0}^{\omega} \alpha_{1}(t) d t+\frac{x_{2}}{\omega} \int_{0}^{\omega} \beta_{1}(t) d t+\sum_{j=1}^{m} \frac{x_{1}}{\omega} \int_{0}^{\omega} p_{1 j}(t) e^{-q_{1 j}(t) x_{1}} d t \\
-\frac{x_{2}}{\omega} \int_{0}^{\omega} \alpha_{2}(t) d t+\frac{x_{1}}{\omega} \int_{0}^{\omega} \beta_{2}(t) d t+\sum_{j=1}^{m} \frac{x_{2}}{\omega} \int_{0}^{\omega} p_{2 j}(t) e^{-q_{2 j}(t) x_{2}} d t
\end{array}\right)
$$


In view of (38), we have

$$
\begin{aligned}
& \max _{0 \leq t \leq \omega}\left|Q N\left(x_{1}, x_{2}\right)_{1}^{T}\right| \\
& =\max _{0 \leq t \leq \omega} \mid-\frac{x_{1}}{\omega} \int_{0}^{\omega} \alpha_{1}(t) d t+\frac{x_{2}}{\omega} \int_{0}^{\omega} \beta_{1}(t) d t \\
& +\sum_{j=1}^{m} \frac{x_{1}}{\omega} \int_{0}^{\omega} p_{1 j}(t) e^{-q_{1 j}(t) x_{1}} d t \\
& \geq \frac{x_{1}}{\omega} \int_{0}^{\omega} \alpha_{1}(t) d t-\frac{x_{2}}{\omega} \int_{0}^{\omega} \beta_{1}(t) d t \\
& -\sum_{j=1}^{m} \frac{x_{1}}{\omega} \int_{0}^{\omega} p_{1 j}(t) e^{-q_{1 j}(t) x_{1}} d t \\
& \geq \alpha_{1}^{-} x_{1}-\beta_{1}^{+} x_{2}-\sum_{j=1}^{m} \frac{p_{1 j}^{+}}{q_{1 j}^{-}} \cdot q_{1 j}^{-} x_{1} e^{-q_{1 j}^{-} x_{1}} \\
& \geq \alpha_{1}^{-} x_{1}-\beta_{1}^{+} x_{2}-\sum_{j=1}^{m} \frac{p_{1 j}^{+}}{q_{1 j}^{-}} \\
& \max _{0 \leq t \leq \omega}\left|Q N\left(x_{1}, x_{2}\right)_{2}^{T}\right| \\
& =\max _{0 \leq t \leq \omega} \mid-\frac{x_{2}}{\omega} \int_{0}^{\omega} \alpha_{2}(t) d t+\frac{x_{1}}{\omega} \int_{0}^{\omega} \beta_{2}(t) d t \\
& +\sum_{j=1}^{m} \frac{x_{2}}{\omega} \int_{0}^{\omega} p_{2 j}(t) e^{-q_{2 j}(t) x_{2}} d t
\end{aligned}
$$

$$
\begin{aligned}
& \geq \frac{x_{2}}{\omega} \int_{0}^{\omega} \alpha_{2}(t) d t-\frac{x_{1}}{\omega} \int_{0}^{\omega} \beta_{2}(t) d t \\
& \quad-\sum_{j=1}^{m} \frac{x_{2}}{\omega} \int_{0}^{\omega} p_{2 j}(t) e^{-q_{2 j}(t) x_{2}} d t \\
& \geq \alpha_{2}^{-} x_{2}-\beta_{2}^{+} x_{1}-\sum_{j=1}^{m} \frac{p_{2 j}^{+}}{q_{2 j}^{-}} \cdot q_{2 j}^{-} x_{2} e^{-q_{2 j}^{-} x_{2}} \\
& \geq \alpha_{2}^{-} x_{2}-\beta_{2}^{+} x_{1}-\sum_{j=1}^{m} \frac{p_{2 j}^{+}}{q_{2 j}^{-}}
\end{aligned}
$$

Equations in (39), together with (37), imply that

$$
\begin{aligned}
& \left\|Q N\left(x_{1}, x_{2}\right)^{T}\right\|_{X} \\
& \geq \frac{1}{2}\left[\max _{0 \leq t \leq \omega}\left|Q N\left(x_{1}, x_{2}\right)_{1}^{T}\right|+\max _{0 \leq t \leq \omega}\left|Q N\left(x_{1}, x_{2}\right)_{2}^{T}\right|\right] \\
& \geq \frac{1}{2}\left[\left(\alpha_{1}^{-}-\beta_{2}^{+}\right) x_{1}+\left(\alpha_{2}^{-}-\beta_{1}^{+}\right) x_{2}-\sum_{j=1}^{m}\left(\frac{p_{1 j}^{+}}{q_{1 j}^{-}}+\frac{p_{2 j}^{+}}{q_{2 j}^{-}}\right)\right] \\
& \geq \frac{1}{2}\left[\widetilde{D} H^{*}-\sum_{j=1}^{m}\left(\frac{p_{1 j}^{+}}{q_{1 j}^{-}}+\frac{p_{2 j}^{+}}{q_{2 j}^{-}}\right)\right]>0 .
\end{aligned}
$$

Consequently, condition (ii) of Lemma 4 is satisfied.

Furthermore, we define a continuous function $\psi$ : $\operatorname{Dom} L \times[0,1] \rightarrow X$ by

$$
\begin{aligned}
\psi\left(x_{1}, x_{2}, \mu\right)= & \mu\left(\begin{array}{c}
-\left(\alpha_{1}^{-}-\beta_{2}^{+}\right) x_{1} \\
-\left(\alpha_{2}^{-}-\beta_{1}^{+}\right) x_{2}
\end{array}\right)+(1-\mu) \\
& \cdot\left(\begin{array}{c}
-\frac{x_{1}}{\omega} \int_{0}^{\omega} \alpha_{1}(t) d t+\frac{x_{2}}{\omega} \int_{0}^{\omega} \beta_{1}(t) d t+\sum_{j=1}^{m} \frac{x_{1}}{\omega} \int_{0}^{\omega} p_{1 j}(t) e^{-q_{1 j}(t) x_{1}} d t \\
-\frac{x_{2}}{\omega} \int_{0}^{\omega} \alpha_{2}(t) d t+\frac{x_{1}}{\omega} \int_{0}^{\omega} \beta_{2}(t) d t+\sum_{j=1}^{m} \frac{x_{2}}{\omega} \int_{0}^{\omega} p_{2 j}(t) e^{-q_{2 j}(t) x_{2}} d t
\end{array}\right) .
\end{aligned}
$$

When $\left(x_{1}, x_{2}\right)^{T} \in \partial \Omega \bigcap \operatorname{Ker} L$ and $\mu \in[0,1],\left(x_{1}(t), x_{2}(t)\right)^{T}$ is a constant vector $\left(x_{1}, x_{2}\right)^{T}$ in $R^{2}$ with $\left\|\left(x_{1}, x_{2}\right)^{T}\right\|_{X}=$ $\max \left\{\left|x_{1}\right|_{\infty},\left|x_{2}\right|_{\infty}\right\}=H^{*}$, then, from (37), we obtain

$$
\begin{aligned}
& \left\|\psi\left(x_{1}, x_{2}, \mu\right)\right\|_{X} \\
& \quad \geq \frac{1}{2}\left\{\mid-\mu\left(\alpha_{1}^{-}-\beta_{2}^{+}\right) x_{1}+(1-\mu)\right.
\end{aligned}
$$

$$
\begin{gathered}
\cdot\left[-\frac{x_{1}}{\omega} \int_{0}^{\omega} \alpha_{1}(t) d t+\frac{x_{2}}{\omega} \int_{0}^{\omega} \beta_{1}(t) d t\right. \\
\left.+\sum_{j=1}^{m} \frac{x_{1}}{\omega} \int_{0}^{\omega} p_{1 j}(t) e^{-q_{1 j}(t) x_{1}} d t\right] \mid \\
+\mid-\mu\left(\alpha_{2}^{-}-\beta_{1}^{+}\right) x_{2}+(1-\mu)
\end{gathered}
$$




$$
\begin{aligned}
& \cdot\left[-\frac{x_{2}}{\omega} \int_{0}^{\omega} \alpha_{2}(t) d t+\frac{x_{1}}{\omega} \int_{0}^{\omega} \beta_{2}(t) d t\right. \\
& \left.\left.+\sum_{j=1}^{m} \frac{x_{2}}{\omega} \int_{0}^{\omega} p_{2 j}(t) e^{-q_{2 j}(t) x_{2}} d t\right] \mid\right\} \\
& \geq \frac{1}{2}\left\{\mu\left(\alpha_{1}^{-}-\beta_{2}^{+}\right) x_{1}+(1-\mu) \frac{x_{1}}{\omega} \int_{0}^{\omega} \alpha_{1}(t) d t\right. \\
& -(1-\mu) \frac{x_{2}}{\omega} \int_{0}^{\omega} \beta_{1}(t) d t \\
& -(1-\mu) \sum_{j=1}^{m} \frac{x_{1}}{\omega} \int_{0}^{\omega} p_{1 j}(t) e^{-q_{1 j}(t) x_{1}} d t \\
& +\mu\left(\alpha_{2}^{-}-\beta_{1}^{+}\right) x_{2}+(1-\mu) \frac{x_{2}}{\omega} \int_{0}^{\omega} \alpha_{2}(t) d t \\
& -(1-\mu) \frac{x_{1}}{\omega} \int_{0}^{\omega} \beta_{2}(t) d t \\
& \left.-(1-\mu) \sum_{j=1}^{m} \frac{x_{2}}{\omega} \int_{0}^{\omega} p_{2 j}(t) e^{-q_{2 j}(t) x_{1}} d t\right\} \\
& \geq \frac{1}{2}\left\{\mu\left(\alpha_{1}^{-}-\beta_{2}^{+}\right) x_{1}+(1-\mu) \alpha_{1}^{-} x_{1}\right. \\
& -(1-\mu) \beta_{1}^{+} x_{2}-(1-\mu) \sum_{j=1}^{m} \frac{p_{1 j}^{+}}{\overline{q_{1 j}^{-}}} \\
& +\mu\left(\alpha_{2}^{-}-\beta_{1}^{+}\right) x_{2}+(1-\mu) \alpha_{2}^{-} x_{2} \\
& \left.-(1-\mu) \beta_{2}^{+} x_{1}-(1-\mu) \sum_{j=1}^{m} \frac{p_{2 j}^{+}}{q_{2 j}^{-}}\right\} \\
& \geq \frac{1}{2}\left\{\left(\alpha_{1}^{-}-\beta_{2}^{+}\right) x_{1}+\left(\alpha_{2}^{-}-\beta_{1}^{+}\right) x_{2}\right. \\
& \left.-(1-\mu) \sum_{j=1}^{m}\left(\frac{p_{1 j}^{+}}{q_{1 j}^{-}}+\frac{p_{2 j}^{+}}{q_{2 j}^{-}}\right)\right\} \\
& \geq \frac{1}{2}\left\{\widetilde{D} H^{*}-\sum_{j=1}^{m}\left(\frac{p_{1 j}^{+}}{q_{1 j}^{-}}+\frac{p_{2 j}^{+}}{q_{2 j}^{-}}\right)\right\}>0 \text {. }
\end{aligned}
$$

It follows that

$$
\begin{aligned}
\psi\left(x_{1}, x_{2}, \mu\right) & \neq(0,0)^{T}, \\
\forall\left(x_{1}, x_{2}\right)^{T} & \in \partial \Omega \cap \operatorname{Ker} L, \mu \in[0,1] .
\end{aligned}
$$

Hence, using the homotopy invariance theorem, we obtain

$$
\begin{aligned}
\operatorname{deg} & \left\{Q N, \Omega \cap \operatorname{Ker} L,(0,0)^{T}\right\} \\
= & \operatorname{deg}\left\{\left(-\left(\alpha_{1}^{-}-\beta_{2}^{+}\right) x_{1},-\left(\alpha_{2}^{-}-\beta_{1}^{+}\right) x_{2}\right)^{T},\right. \\
& \left.\Omega \cap \operatorname{Ker} L,(0,0)^{T}\right\} \neq 0 .
\end{aligned}
$$

Condition (iii) of Lemma 4 is also satisfied. Thus, by Lemma 4, we conclude that $L u=N u, u=\left(x_{1}(t), x_{2}(t)\right)^{T}$; that is, (6) has at least one solution in $X$. Then, by Lemma 2 , we immediately obtain that (3) has at least one positive $\omega$ periodic solution. This completes the proof.

Theorem 6. Let $\left(A_{1}\right)-\left(A_{3}\right)$ hold; furthermore, assume that the following conditions are satisfied:

$$
\begin{aligned}
& \left(A_{5}\right) \min \left\{\left(\alpha_{1}^{-}-\beta_{2}^{+}\right)-\sum_{j=1}^{m} 2 p_{1 j}^{+},\left(\alpha_{2}^{-}-\beta_{1}^{+}\right)-\sum_{j=1}^{m} 2 p_{2 j}^{+}\right\}>0, \\
& \left(A_{6}\right) \tau_{i j}(t) \in C^{1}([0, \infty),(0, \infty)) \text { and } \tau_{i j}^{\prime}(t) \leq 0, i=1,2, \\
& j=1,2, \ldots, m .
\end{aligned}
$$

Then (3) has a unique positive $\omega$-periodic solution.

Proof. By Lemma 2, it suffices to prove the uniqueness of positive $\omega$-periodic solutions for system (6). According to Theorem 5, we know that (6) has at least a positive $\omega$-periodic solution $x^{*}(t)=\left(x_{1}^{*}(t), x_{2}^{*}(t)\right)^{T}$ with initial condition (4). Suppose that $x(t)=\left(x_{1}(t), x_{2}(t)\right)^{T}$ is an arbitrary positive $\omega$-periodic solution of (6) with initial condition (4). Then it follows from (6) that

$$
\begin{aligned}
\frac{d}{d t}\left(x_{1}(t)-x_{1}^{*}(t)\right)=- & \alpha_{1}(t)\left[x_{1}(t)-x_{1}^{*}(t)\right] \\
+ & \beta_{1}(t)\left[x_{2}(t)-x_{2}^{*}(t)\right]+\sum_{j=1}^{m} p_{1 j}(t) \\
\cdot & {\left[x_{1}\left(t-\tau_{1 j}(t)\right) e^{-q_{1 j}(t) x_{1}\left(t-\tau_{1 j}(t)\right)}\right.} \\
& \left.-x_{1}^{*}\left(t-\tau_{1 j}(t)\right) e^{-q_{1 j}(t) x_{1}^{*}\left(t-\tau_{1 j}(t)\right)}\right] .
\end{aligned}
$$

Calculating the upper-right derivative, we have

$$
\begin{aligned}
& \frac{d^{+}}{d t}\left|x_{1}(t)-x_{1}^{*}(t)\right| \\
& \leq-\alpha_{1}^{-}\left|x_{1}(t)-x_{1}^{*}(t)\right|+\beta_{1}^{+}\left|x_{2}(t)-x_{2}^{*}(t)\right| \\
& +\sum_{j=1}^{m} p_{1 j}^{+} \mid x_{1}\left(t-\tau_{1 j}(t)\right) \\
& \cdot e^{-q_{1 j}(t) x_{1}\left(t-\tau_{1 j}(t)\right)}-x_{1}^{*}\left(t-\tau_{1 j}(t)\right) \\
& \cdot e^{-q_{1 j}(t) x_{1}^{*}\left(t-\tau_{1 j}(t)\right)} \mid .
\end{aligned}
$$


For $y=x e^{-r x}, r>0$ is a real number, $0<a \leq x \leq b$, and by mean value theorem, we have

$$
\begin{aligned}
\left|b e^{-r b}-a e^{-r a}\right| & =\left|a e^{-r a}-b e^{-r b}\right| \\
& =\left|a e^{-r a}-a e^{-r b}+a e^{-r b}-b e^{-r b}\right| \\
& \leq\left|-a r e^{-r \xi}\right||b-a|+\left|e^{-r b}\right||b-a| \\
& =\left|a r e^{-r a} \cdot e^{-r(\xi-a)}\right||b-a|+\left|e^{-r b}\right||b-a| \\
& \leq 2|b-a|,
\end{aligned}
$$

where $a<\xi<b$. Thus, for any fixed $j=1,2, \ldots, m$, we also have

$$
\begin{aligned}
& \mid x_{1}^{*}\left(t-\tau_{1 j}(t)\right) e^{-q_{1 j(t)} x_{1}^{*}\left(t-\tau_{1 j}(t)\right)} \\
& -x_{1}\left(t-\tau_{1 j}(t)\right) e^{-q_{1 j}(t) x_{1}\left(t-\tau_{1 j}(t)\right)} \mid \\
& \quad \leq 2\left|x_{1}\left(t-\tau_{1 j}(t)\right)-x_{1}^{*}\left(t-\tau_{1 j}(t)\right)\right| .
\end{aligned}
$$

Hence,

$$
\begin{aligned}
& \frac{d^{+}}{d t}\left|x_{1}(t)-x_{1}^{*}(t)\right| \\
& \leq-\alpha_{1}^{-}\left|x_{1}(t)-x_{1}^{*}(t)\right|+\beta_{1}^{+}\left|x_{2}(t)-x_{2}^{*}(t)\right| \\
& \quad+\sum_{j=1}^{m} 2 p_{1 j}^{+}\left|x_{1}\left(t-\tau_{1 j}(t)\right)-x_{1}^{*}\left(t-\tau_{1 j}(t)\right)\right| .
\end{aligned}
$$

Similarly, we have

$$
\begin{aligned}
& \frac{d^{+}}{d t}\left|x_{2}(t)-x_{2}^{*}(t)\right| \\
& \leq-\alpha_{2}^{-}\left|x_{2}(t)-x_{2}^{*}(t)\right|+\beta_{2}^{+}\left|x_{1}(t)-x_{1}^{*}(t)\right| \\
& \quad+\sum_{j=1}^{m} 2 p_{2 j}^{+}\left|x_{2}\left(t-\tau_{2 j}(t)\right)-x_{2}^{*}\left(t-\tau_{2 j}(t)\right)\right| .
\end{aligned}
$$

We define a Lyapunov function $V(\cdot)$ by

$$
\begin{aligned}
V(t)= & V\left(x_{1}, x_{2}\right)(t) \\
= & \sum_{i=1}^{2}\left|x_{i}(t)-x_{i}^{*}(t)\right| \\
& +\sum_{j=1}^{m} \int_{t-\tau_{1 j}(t)}^{t} 2 p_{1 j}^{+}\left|x_{1}(s)-x_{1}^{*}(s)\right| d s \\
& +\sum_{j=1}^{m} \int_{t-\tau_{2 j}(t)}^{t} 2 p_{2 j}^{+}\left|x_{2}(s)-x_{2}^{*}(s)\right| d s,
\end{aligned}
$$

for $t>0$, and by virtue of (49), (50), and assumption $\left(A_{6}\right)$, we get

$$
\begin{aligned}
& \frac{d^{+} V(t)}{d t} \\
& \leq-\left(\alpha_{1}^{-}-\beta_{2}^{+}\right)\left|x_{1}(t)-x_{1}^{*}(t)\right| \\
& -\left(\alpha_{2}^{-}-\beta_{1}^{+}\right)\left|x_{2}(t)-x_{2}^{*}(t)\right| \\
& +\sum_{j=1}^{m} 2 p_{1 j}^{+}\left|x_{1}\left(t-\tau_{1 j}(t)\right)-x_{1}^{*}\left(t-\tau_{1 j}(t)\right)\right| \\
& +\sum_{j=1}^{m} 2 p_{2 j}^{+}\left|x_{2}\left(t-\tau_{2 j}(t)\right)-x_{2}^{*}\left(t-\tau_{2 j}(t)\right)\right| \\
& +\sum_{j=1}^{m} 2 p_{1 j}^{+}\left|x_{1}(t)-x_{1}^{*}(t)\right|+\sum_{j=1}^{m} 2 p_{2 j}^{+}\left|x_{2}(t)-x_{2}^{*}(t)\right| \\
& -\sum_{j=1}^{m} 2 p_{1 j}^{+}\left|x_{1}\left(t-\tau_{1 j}(t)\right)-x_{1}^{*}\left(t-\tau_{1 j}(t)\right)\right| \\
& \cdot\left(1-\tau_{1 j}^{\prime}(t)\right) \\
& -\sum_{j=1}^{m} 2 p_{2 j}^{+}\left|x_{2}\left(t-\tau_{2 j}(t)\right)-x_{2}^{*}\left(t-\tau_{2 j}(t)\right)\right| \\
& \cdot\left(1-\tau_{2 j}^{\prime}(t)\right) \\
& \leq-\left[\left(\alpha_{1}^{-}-\beta_{2}^{+}\right)-\sum_{j=1}^{m} 2 p_{1 j}^{+}\right]\left|x_{1}(t)-x_{1}^{*}(t)\right| \\
& -\left[\left(\alpha_{2}^{-}-\beta_{1}^{+}\right)-\sum_{j=1}^{m} 2 p_{2 j}^{+}\right]\left|x_{2}(t)-x_{2}^{*}(t)\right| \\
& \leq-\min \left\{\left(\alpha_{1}^{-}-\beta_{2}^{+}\right)-\sum_{j=1}^{m} 2 p_{1 j}^{+},\left(\alpha_{2}^{-}-\beta_{1}^{+}\right)-\sum_{j=1}^{m} 2 p_{2 j}^{+}\right\} \\
& \cdot\left(\left|x_{1}(t)-x_{1}^{*}(t)\right|+\left|x_{2}(t)-x_{2}^{*}(t)\right|\right) \text {. }
\end{aligned}
$$

According to $\left(A_{5}\right)$,

$$
\min \left\{\left(\alpha_{1}^{-}-\beta_{2}^{+}\right)-\sum_{j=1}^{m} 2 p_{1 j}^{+},\left(\alpha_{2}^{-}-\beta_{1}^{+}\right)-\sum_{j=1}^{m} 2 p_{2 j}^{+}\right\}>0
$$

and it follows that

$$
\frac{d^{+} V(t)}{d t} \leq 0, \quad t>0 .
$$

Hence, we obtain

$$
V(t) \leq V(0), \quad \forall t>0
$$


So

$$
\begin{aligned}
\sum_{i=1}^{2}\left|x_{i}(t)-x_{i}^{*}(t)\right| \leq V(t) \leq V(0) \\
=\sum_{i=1}^{2}\left|x_{i}(0)-x_{i}^{*}(0)\right|=0, \quad \forall t>0 .
\end{aligned}
$$

In view of (56) and periodicity of $x_{i}(t)-x_{i}^{*}(t)$, we have

$$
x_{i}(t)-x_{i}^{*}(t)=0, \quad \forall t>0, i=1,2 .
$$

Then by Lemma 2 , we conclude that (3) has a unique positive $\omega$-periodic solution. This completes the proof.

\section{Conclusion}

In this paper, a class of Nicholson-type systems with impulses and delays are investigated. We conquer the difficulty of coexistence of impulsive and delay factors in a dynamic system and give some results of the existence and uniqueness of positive periodic solutions. The results in this paper extend some earlier works reported in the literature. Moreover, our results are easy to test and important in applications of periodic oscillatory delayed Nicholson-type systems with impulsive control.

\section{Acknowledgments}

This work is supported by the National Natural Science Foundation of China (Grant no. 11171374) and the Scientific Research Fund of Shandong Provincial of China (Grant no. ZR2011AZ001).

\section{References}

[1] W. S. Gurney, S. P. Blythe, and R. M. Nisber, "Nicholson's blowflies revisited," Nature, vol. 287, no. 5777, pp. 17-21, 1980.

[2] I. Győri and G. Ladas, Oscillation Theory of Delay Differential Equations with Applications, Clarendon, Oxford, UK, 1991.

[3] K. Gopalsamy, Stability and Oscillation in Delay Differential Equations of Population Dynamics, Kluwer Academic Publishers, Boston, Mass, USA, 1992.

[4] Y. Kuang, Delay Differential Equations with Applications in Population Dynamics, Academic Press, Boston, Mass, USA, 1993.

[5] F. Brauer and C. Castillo-Chávez, Mathematical Models in Population Biology and Epidemiology, Springer, New York, NY, USA, 2001.

[6] M. Kot, Elements of Mathematical Ecology, Cambridge University Press, Cambridge, UK, 2001.

[7] S. H. Saker and S. Agarwal, "Oscillation and global attractivity in a periodic Nicholson's blowflies model," Mathematical and Computer Modelling, vol. 35, no. 7-8, pp. 719-731, 2002.

[8] Y. Chen, "Periodic solutions of delayed periodic Nicholson's blowflies models," The Canadian Applied Mathematics Quarterly, vol. 11, no. 1, pp. 23-28, 2003.

[9] W.-T. Li and Y.-H. Fan, "Existence and global attractivity of positive periodic solutions for the impulsive delay Nicholson's blowflies model," Journal of Computational and Applied Mathematics, vol. 201, no. 1, pp. 55-68, 2007.
[10] J. Li and C. Du, "Existence of positive periodic solutions for a generalized Nicholson's blowflies model," Journal of Computational and Applied Mathematics, vol. 221, no. 1, pp. 226-233, 2008.

[11] W. Chen and B. Liu, "Positive almost periodic solution for a class of Nicholson's blowflies model with multiple time-varying delays," Journal of Computational and Applied Mathematics, vol. 235, no. 8, pp. 2090-2097, 2011.

[12] L. Berezansky, L. Idels, and L. Troib, "Global dynamics of Nicholson-type delay systems with applications," Nonlinear Analysis. Real World Applications, vol. 12, no. 1, pp. 436-445, 2011.

[13] W. Wang, L. Wang, and W. Chen, "Existence and exponential stability of positive almost periodic solution for Nicholson-type delay systems," Nonlinear Analysis. Real World Applications, vol. 12, no. 4, pp. 1938-1949, 2011.

[14] B. Liu, "The existence and uniqueness of positive periodic solutions of Nicholson-type delay systems," Nonlinear Analysis. Real World Applications, vol. 12, no. 6, pp. 3145-3151, 2011.

[15] V. Lakshmikantham, D. D. Bainnov, and P. S. Simeonov, Theory of Impulsive Differential Equations, World Scientific, Singapore, 1989.

[16] D. D. Bainov and P. S. Simeonov, Theory of Impulsive Differential Equations: Periodic Solutions and Applications, Longman, Harlow, UK, 1993.

[17] A. M. Samoilenko and N. A. Perestyuk, Differential Equations With Impulsive Effect, World Scientific, Singapore, 1995.

[18] J. Yan, "Existence and global attractivity of positive periodic solution for an impulsive Lasota-Wazewska model," Journal of Mathematical Analysis and Applications, vol. 279, no. 1, pp. 111120, 2003.

[19] R. E. Gaines and J. L. Mawhin, Coincidence Degree and Nonlinear Differential Equations, Springer, Berlin, Germany, 1977. 


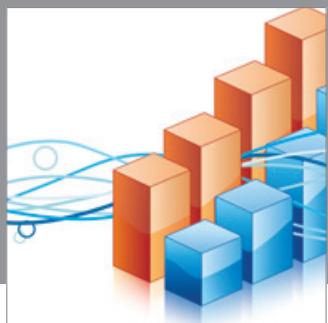

Advances in

Operations Research

mansans

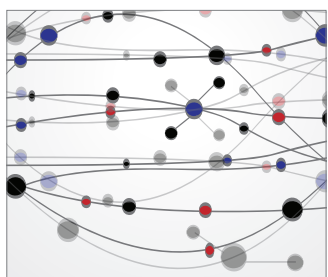

The Scientific World Journal
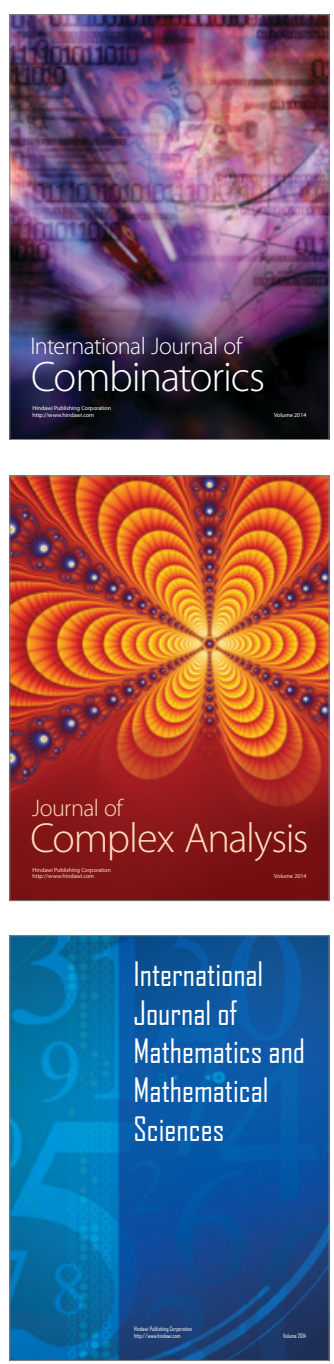
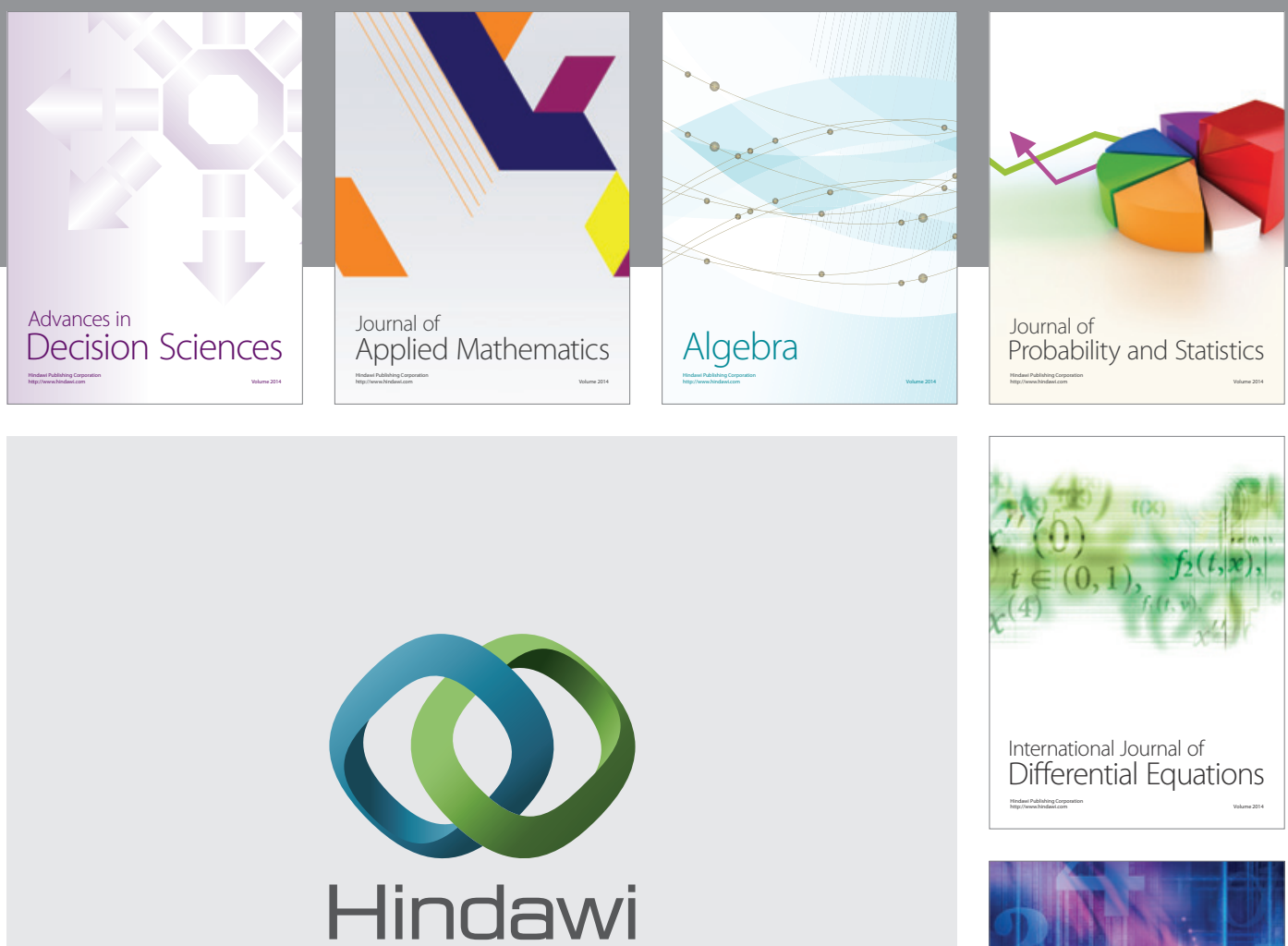

Submit your manuscripts at http://www.hindawi.com
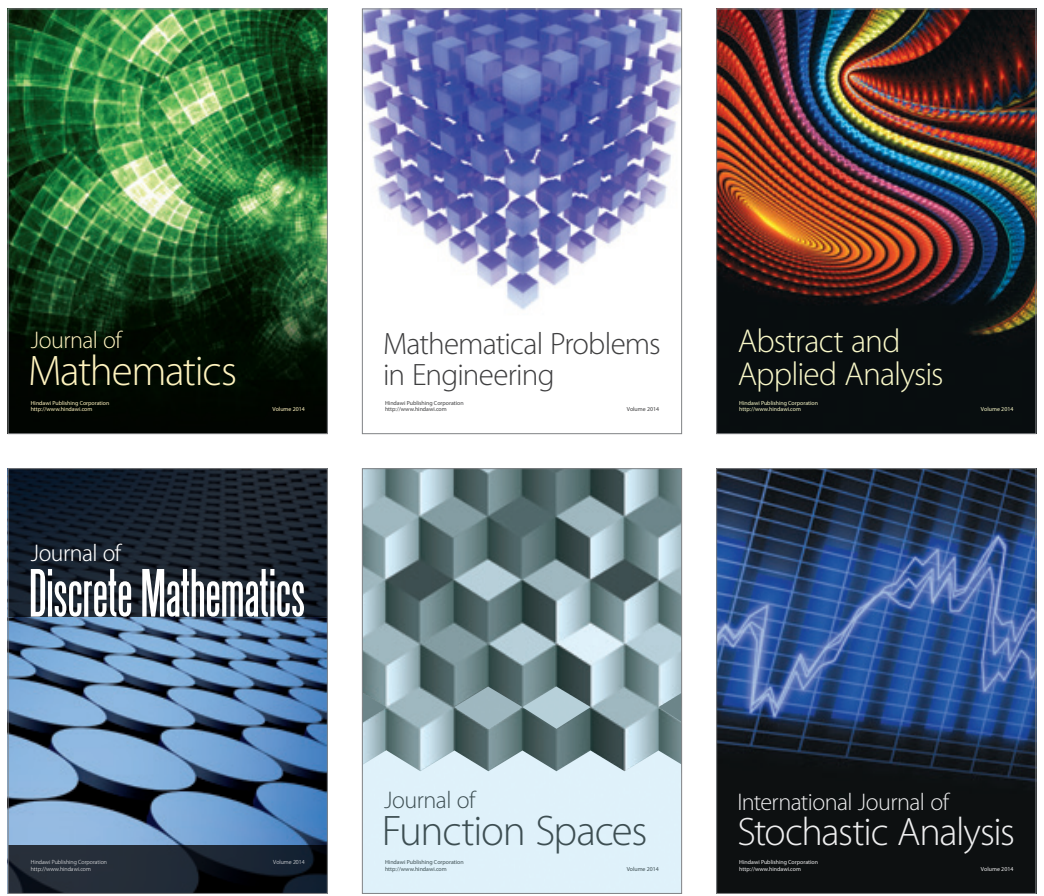

Journal of

Function Spaces

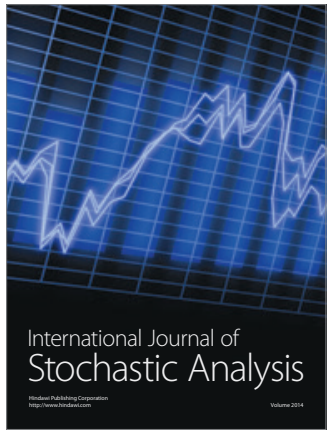

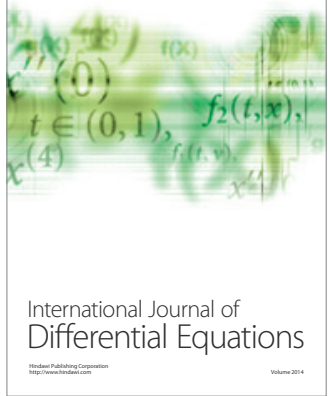
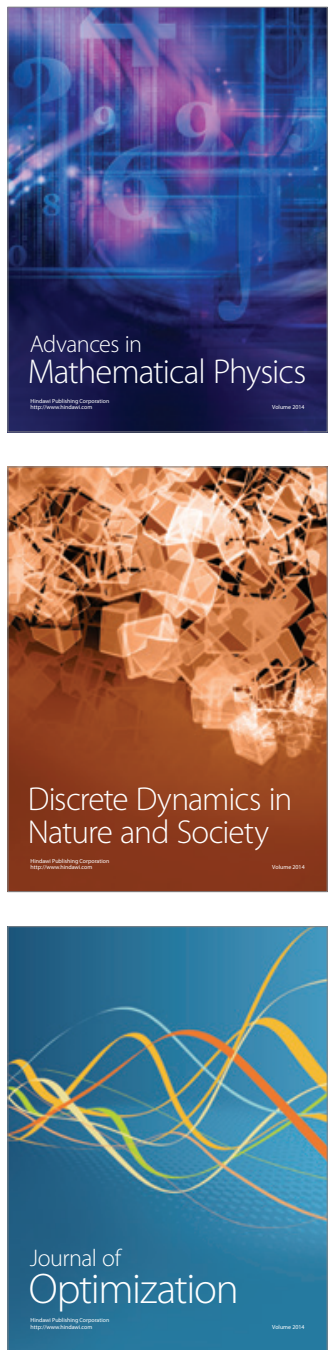